\title{
La formación profesional y sus ámbitos de acción: el caso del Taller de Integración Curricular II
}

\author{
Christophe Albaladejo ${ }^{1}$, Ramón Cieza², Alejandra Moreyra ${ }^{3}$, María Celina \\ Bernal $^{2}$ y Mariano Dell' Aquila ${ }^{2}$
}

\section{Resumen}

La Facultad de Ciencias Agrarias y Forestales (Universidad Nacional de La Plata) es una de las pocas de Argentina que dicta las carreras de ingeniería forestal y de ingeniería agronómica en la misma unidad académica. Desde su creación, la facultad ha apostado a la formación de un profesional de perfil generalista que pueda desenvolverse en la variedad de contextos de nuestro país. Esta característica expone un importante desafío: formar ingenieros aptos para trabajar en un mundo profesional muy diverso y hasta fragmentado, constituido por distintos "modelos de desarrollo" que requieren diferentes "paradigmas tecnológicos y sociales". Este artículo presenta los avances en cuanto a la implementación de un Taller de Integración Curricular desarrollado en el último año de ambas carreras con el objetivo de aportar a la formación de profesionales preparados para trabajar en el sentido planteado.

Palabras clave: Modelos de desarrollo-Paradigmas tecnológicos-Competencias - Integración Curricular - Ingeniería.

1 UNLP Facultad de Ciencias Agrarias y Forestales e INRA UMR

2 UNLP. Facultad de Ciencias Agrarias y Forestales

3 UNLP Facultad de Ciencias Agrarias y Forestales e INTA IPAF Instituto

de Investigación y Desarrollo Tecnológico para la Agricultura Familiar-Región

Pampeana 


\section{Abstract}

The Faculty of Agricultural and Forestry Sciences (National University of La Plata) is one of the few in Argentina which dictates forestry and agricultural engineering in the same academic unit. Since its inception, the faculty has committed to the formation of a generalist professional able to approach the variety of working contexts in our country. This feature presents a major challenge: to form engineers capable of working in a diverse and fragmented professional world, consisting of various "development models" that require different "technological and social paradigms." This paper presents the progress in the implementation of a Curriculum Integration Workshop developed in the last year of both carriers with the aim of contributing to train professionals prepared to work in the stated direction.

Key words: Development models - Technological Paradigms - Skills - Curriculum Integration - Engineerin. 


\section{Introducción}

Con el cambio en el plan de estudios, en el año 2005 se implementan para las carreras de Ingeniería Agronómica e Ingeniería Forestal de la Universidad Nacional de La Plata espacios curriculares a los fines de generar integración de los contenidos. Entre ellos, el Taller de Integración Curricular II propone tener contactos con diferentes actores del mundo profesional y las heterogeneidades existentes. Frente a esto, los estudiantes aprenden a saber reconocer y valorar la diversidad en las formas de practicar la agricultura y reflexionan sobre sus desempeños en estos diversos contextos, la validez de los conocimientos adquiridos y sobre sus identidades profesionales. Un viaje de estudio de una semana a una provincia (que ha sido hasta la fecha Entre Ríos para Agronomía y Misiones para Ingeniería Forestal) con docentes de diversas cátedras de las carreras, permite avanzar aún más en la reflexión sobre la noción de "contexto", en el arte de la ingeniería y sobre la diversidad de formas de practicar la agricultura y las yuxtaposiciones entre formas de agricultura e interrelaciones ad hoc en el territorio.

Este trabajo presenta los avances de esta reflexión a propósito de la formación y de la ingeniería a partir de la experiencia de tres años de implementación del Taller con aproximadamente cien estudiantes por año. Por otra parte reflexiona sobre la visión de la ingeniería sobre la cual reposa este taller, las percepciones en cuanto a la integración y su rol en los nuevos planes de las carreras.

\section{La ingeniería y el contexto de acción}

Dado su carácter generalista y su consecuente formación de profesionales para desarrollar su actividad en los distintos puntos del país, saber tomar en cuenta la diversidad de la actividad agropecuaria ha sido un desafío de larga data para la Facultad de Ciencias Agrarias y Forestales, la más antigua del país en agronomía (año de creación 1883). Sin embargo, pese a la alta diversidad de medios y de sistemas de producción en los cuales ha sido susceptible de desempeñarse el futuro profesional de la facultad, se consideraba implícitamente, como en todas la otras facultades del país, que existía un único modelo de modernización de la actividad. De hecho se consideraba que los mismos conocimientos científicos y las mismas tecnologías podían ser adaptados a lo largo y a lo ancho del país y más aún a todos los productores de una misma región. De esta forma se posicionaba la formación desde la unicidad si no del mundo agropecuario y forestal por lo menos de su perspectiva de cambio, y en especial, en cuanto a la búsqueda de un horizonte de desarrollo. Así la facultad podía transmitir conocimientos genéricos universalmente válidos y el arte del profesional, que era desarrollado "en la práctica", era el de 
saber adaptar e integrar estos conocimientos en función de la situación de acción. En síntesis, el profesional estaba formado en base a una ciencia universalista, unificada y descontextualizada y las situaciones de acción no estaban consideradas dignas del abordaje científico, sino como relevando más bien del arte de la práctica, de la experiencia personal y entonces de un arte de "saber arreglarse" elaborado en una cierta "soledad" del profesional, o a lo mejor en redes informales de pares, pero siempre sin una construcción conceptual y sistematizadora aportada por su formación en la facultad. A lo sumo durante la carrera se organizaban salidas "a campo", únicamente con fines ilustrativos de lo visto en el aula y sin la aplicación y construcción de un análisis contextual.

La solución para acercar la enseñanza a las situaciones concretas de acción de los profesionales empezó a plantearse desde la idea de "integración". Recién en los últimos quince años, la necesidad de la integración de conocimientos y su vinculación con la formación de ingenieros comenzó a discutirse en las facultades de agronomía y de ciencias forestales. La falta de integración de los conocimientos adquiridos en los diferentes cursos y la certeza de que su construcción emergía de y volvía a realidades concretas, tomaban fuerza en el diagnóstico para el cambio de plan de estudios. Díaz Maynard y Vellani (2008) plantean claramente esta problemática común para las carreras de ingeniería agronómica de Argentina y Uruguay: "El estudiante transcurría su tiempo de estudio en un ambiente ajeno a la realidad, a la problemática agronómica, sin oportunidad de integrar conocimientos, ni de desarrollar habilidades, ni de complementar teoría y práctica, ni de recibir estímulos de la realidad productiva, ni de convivir con los agentes del medio productivo para conocerlos y entenderlos". Es así que en la modificación de los planes de estudio en las distintas facultades de agronomía del país, se comienzan a atender las problemáticas antes descriptas, aunque con diferencias temporales y en cuanto a la forma de implementación. En el caso de la FCAyF-UNLP, se realiza una modificación en los planes de estudio en el año 1999 y una posterior en el año 2006. En esta última se incorporan instancias de integración, entre ellas el taller de integración curricular II (TIC II) al término de la carrera.

\section{Los espacios de integración}

En su origen, el nuevo Plan de Estudios asume que tres espacios curriculares deben constituir un eje articulador e integrador para la formación del estudiante de las carreras de Ingeniería Agronómica e Ingeniería Forestal. Uno inicial (o propedéutico) el cual ya estaba en el plan de estudios aprobado en el año 1999 (Introducción a las Ciencias Agrarias y Forestales) y luego dos talleres de integración en tercero y quinto año. Estos últimos espacios curriculares surgieron con una 
función específica, la de integrar conocimientos trabajados por los estudiantes en espacios disciplinares de tramos formativos previos, sin incorporación de contenidos o conceptos nuevos. El enfoque de sistemas se planteó como un eje estructurante para los espacios de integración iniciales.

En la materia de primer año se supone que el estudiante aprende y reconoce los principales componentes de las actividades agropecuarias y forestales pero aún carece de un conocimiento en profundidad de los mismos o de las diferentes interacciones que éstos puedan comprender. En este espacio curricular se comienza a utilizar el enfoque de sistemas en una escala de predio y de esta manera se intenta acercarle al estudio y a la comprensión de las acciones del productor. En esta etapa se analizan los elementos del sistema (sistema de cultivo o sistema ganadero) aplicado a esta escala (límites, componentes, interacciones, entradas y salidas) y se genera un estudio más profundo de estos elementos. Esta instancia se complementa con un análisis de un sistema de producción al cual los estudiantes visitan en dos oportunidades.

En la posterior instancia, al finalizar tercer año (Taller de Integración Curricular I), se busca integrar contenidos de las asignaturas de ciencias básicas de los tres primeros años de las carreras, como así también situar al estudiante en lo que le resta transitar en su formación a partir de la generación de interrogantes que deberán responderse en las asignaturas de ciencias aplicadas de los dos últimos años de la carrera. Este espacio curricular se centra principalmente a nivel de agroecosistema (aplicado a nivel del sistema de producción, sistema de explotación o sistema de actividad de la explotación). Los estudiantes retoman explícitamente el empleo del enfoque de sistemas. Primero lo aplican al estudio de un individuo (vegetal y animal) incrementando la escala de análisis a la de población y comunidad. El enfoque de sistemas internalizado y aplicado por el estudiante tiene como objeto principal "la realidad en la cual está inserto". Es decir, la adquisición de conocimiento acerca del enfoque de sistemas (conocimiento declarativo) y la capacidad de emplearlo en la praxis diaria (conocimiento procedimental) realizándolo a partir del estudio y análisis en un contexto real.

Por último, el Taller de Integración Curricular II constituye un espacio curricular realizado al finalizar las carreras. Se basa en el concepto de "modelos de desarrollo" y también en la idea de "co-presencia de diferentes modelos en el mismo territorio". El "Modelo" per se se puede representar como un conjunto de actores, objetos y conocimientos que constituyen un cierto tipo de agricultura (campesina, familiar, convencional, empresarial, industrial, financiera). En particular es la manifestación de un "sistema de conocimientos y de información", enfoque que podemos articular con los aportes de la asignatura "Extensión rural" vista con anterioridad. También cada modelo tiene una "base social" constituida en particular por agricultores y sus familias que llevan adelante preferentemente ciertos tipos de sistema de producción; si bien la correspondencia no es automática entre tipos de sistemas 
de producción y modelos de desarrollo ya que un modelo de desarrollo como por ejemplo la agricultura familiar comporta una gran variedad de sistemas de producción. Esto conduce a reforzar la movilización del enfoque sistémico por un lado, y trascenderlo por el otro.

La reflexión sobre la "co-presencia" (articulaciones, contradicciones, enfrentamientos, luchas, sinergias) entre estos modelos pasa por el concepto de "territorio". Se trata del territorio conceptualizado desde la geografía social, ya no desde un concepto sistémico como lo es el de "sistema agrario". Es una dimensión importante de la profesionalidad de los ingenieros forestales y agrónomos ya que intenta prepararles para un mundo hecho de negociaciones, articulaciones y conflictos entre diversos actores que pugnan por la preponderancia de los diferentes modelos de desarrollo que conforman.

En este taller se trabaja algo en común con los anteriores espacios de integración: la capacidad de observar en contexto y adaptar los conocimientos de la carrera a la contingencia de las situaciones de acción. La idea de las ingenierías como "ciencias de la acción en contexto" desde donde se puede interpretar la realidad e integrar conceptos, es la base de las tres asignaturas. Si bien el concepto de sistema resulta central, se introducen otros conceptos integradores, es decir, categorías de análisis con capacidad para englobar los conocimientos adquiridos por los alumnos en los cursos previos. Tales conceptos, como "modelos de desarrollo" y "territorio", cuentan con un gran potencial como organizadores de los procesos cognitivos que aporten a una formación más integral de los estudiantes de Ingeniería Agronómica e ingeniería Forestal.

La integración de los conocimientos remite inmediatamente, en el caso de las ingenierías, a los aspectos contextuales de la acción. En efecto hay dos formas de integrar. Se puede integrar con un objetivo de conocimiento disciplinario y en este caso se trata de producir un "objeto" (científico) distinto, o sea una nueva disciplina, cada disciplina teniendo un "objeto" propio. La acción siempre es particular y singular, única y "microcontextualizada". Es por eso que la integración en este caso no intenta discernir un "objeto científico" en el sentido de una representación de la realidad que se pueda encontrar en otro lugar y en otro momento. La integración que se necesita en el caso de una perspectiva de acción, intenta formar una representación eficaz en relación a la situación planteada, situación que a la vez requiere una intervención y es producto de muchas intervenciones anteriores. Por esa razón se trata de una representación efímera y contingente, única e irreproducible y por eso ha sido relegada durante muchos años a las habilidades singulares y personales de los individuos profesionales en acción. Si bien las situaciones de acción no son entonces modelizables, esta segunda forma de integración recurre a un enfoque de ciencia de la ingeniería o sea de una representación de la realidad en vistas a la acción y para tal efecto, reposa sobre una epistemología constructivista. Un enfoque constructivista hace modelos 
de la realidad concebidos contextualmente en función de la intención de acción del modelizador y no bajo la pretensión de independencia de uno y otro. Autores como Jean-Louis Lemoigne (1990) nos recuerdan que la ingeniería, contrariamente a la tendencia general de las facultades que las enseñan basadas en enfoques positivistas, nace con una epistemología constructivista junto con pensadores como Leonardo $\mathrm{Da}$ Vinci o Giambatista Vico. La ingeniería debería ser la ciencia para la acción y entonces una ciencia del contexto, contrariamente a las ciencias positivistas.

\section{Propuesta pedagógica, implementación y resultados}

El posicionamiento de base del Taller es el de la diferenciación de los paradigmas tecnológicos en juego en el agro y en los mundos forestales. Consecuentemente, la postura adoptada es la de considerar que pese a este proceso de fragmentación del campo profesional, sigue existiendo un solo tipo de profesional, pero con capacidades para reconocer los diferentes ámbitos de acción y conceptualizar en consecuencia su práctica profesional. En función de esto, el objetivo del taller TIC II es que los estudiantes movilicen los conocimientos adquiridos durante su carrera en función de los contextos tecnológicos en los cuales van a tener que intervenir. De esta manera se propone que se detecten las diferencias en los ámbitos de intervención, movilicen diferencialmente sus conocimientos de base, y realicen esta detección de acuerdo a una visión global de la dinámica del sector forestal o agropecuario. Por otra parte se busca que caractericen el funcionamiento de un contexto tecnológico para poder recrear su desempeño profesional en términos de a) adecuar los objetivos del diagnóstico b) realizar el diagnóstico en función de las metodologías o modalidades posibles o deseables en este contexto y c) seleccionar, adaptar y saber cómo completar la base de conocimientos adquiridos durante la carrera. En relación a la movilización de los conocimientos adquiridos el estudiante deberá saber seleccionarlos, entendiendo que todos los conocimientos adquiridos no son aptos en cualquier contexto tecnológico y entonces saber buscar con criterio la base de esos conocimientos y evaluar la pertinencia de cada uno. Se busca además una recreación del mismo, ya que un conocimiento adquirido puede ser movilizado de varias maneras en un diagnóstico según las interacciones surgidas en contexto. Por otra parte resulta central identificar los centros de documentación, de investigación, de información, donde el futuro profesional pueda encontrar otros conocimientos que le hacen o harán falta en un contexto dado, considerando que en la facultad ha construido una integralidad del bagaje cognitivo necesario para trabajar en todos los contextos socio-tecnológicos presentes en Argentina.

El curso se realiza bajo la modalidad de taller, a partir de la interacción con actores invitados, la discusión en grupos a partir de consignas dirigidas por el cuerpo docente y la posterior puesta en común en plenario. El taller se inicia con 
una clase teórica que permita conceptualizar la diversidad del mundo rural y la construcción en forma colectiva de una guía de observación para operar en terreno. La diversidad del sector se profundiza con la invitación de productores y actores correspondientes a distintos modelos de desarrollo (campesino, agricultura familiar y empresarial). Estos testimonios son complementados en algunos casos con videos. Luego, los estudiantes divididos en grupos discuten sobre los diferentes aspectos que les permiten identificar los distintos modelos de desarrollo, y reflexionan sobre modos de abordar el trabajo profesional con estos actores. En esta reflexión sobre la acción profesional en ese contexto, se trabaja en la puesta en práctica de otros conocimientos tomados de otros cursos a lo largo de la carrera. Finalmente, este proceso se plasma en un documento realizado por cada uno de los grupos y se exponen en plenario.

En el marco del taller, también se realiza un viaje de integración que se desarrolla en el lapso de cinco días, donde se interactúa en diferentes realidades con distintos actores del sector socio-tecnológico en cuestión. La idea es disponer de una paleta de casos que permita al estudiante poner en cuestionamiento los conocimientos adquiridos y desarrollar su sentido de análisis situacional en el cuál se movilizan estos conocimientos. Al incorporar el viaje integrador al TIC II, la hipótesis es que -aun con un número grande de estudiantes- un viaje concentrando muchos encuentros con actores del campo en cinco días, permite a los estudiantes experimentar la importancia de la caracterización del contexto socio-técnico de intervención para integrar los conocimientos adquiridos y recreados en tales encuentros, en vista a la acción profesional. Por último se realiza un seminario sobre "profesionalidades", para analizar las distintas maneras de desempeñarse como profesional y construirse una legitimidad. Para ellos se convoca a ingenieros que se desempeñan en distintos ámbitos, a compartir sus experiencias y miradas profesionales con los estudiantes. En esta etapa del taller se analiza y discute el oficio del ingeniero, habilidades y conocimientos necesarios, las redes de vinculación con otros actores (profesionales o no) y las estrategias de acción de acuerdo al posicionamiento profesional y los contextos en que interactúan. En cuanto al marco conceptual con el que se trabaja en este proceso, por un lado, está la gestión de sistemas de conocimientos, la concepción de que en el intercambio con los interlocutores locales y entre los mismos, se movilizan, intercambian y hasta negocian conocimientos diferentes que hacen a la construcción social de los mundos forestales y agropecuarios que se busca comprender a través de esta práctica pedagógica. Este marco teórico también les permite describir la diversidad de la actividad agropecuaria y forestal adoptando una visión amplia de ésta, o sea, abarcando:

- una dimensión económica-productiva

- una dimensión de modos de vida

- una dimensión política y asociativa 
Estas son las tres dimensiones de la actividad humana (Arendt 2004), que han sido tomadas como descriptores de la relación de la actividad agropecuaria-forestal en la construcción del territorio (Albaladejo 2009). La noción de "modelos de desarrollo" es complementaria y se refiere a conjuntos de actores de la producción, de la ciencia y de la técnica, del mundo de los negocios, de las administraciones, entre otros, que tienen tendencia a mantener relaciones fuertes entre sí. De esta manera, definen "mundos sociales" (según la noción de la sociología interaccionista -Strauss 1992-) específicos, en los cuales la actividad agropecuaria y/o forestal cobra características particulares y un modo singular de definir el territorio. Cada modelo de desarrollo genera objetos técnicos y científicos propios, políticas públicas o modos de intervención del Estado específicos, y puja para su consolidación y definición de territorio en articulación o a veces contradicción con otros modelos en co-presencia.

\section{Resultados}

La elaboración de una encuesta anónima que se les entrega a los estudiantes al finalizar el taller nos permite la innovación y adecuación de la propuesta pedagógica en base a la retroalimentación dada por las opiniones de los estudiantes. En base al análisis de los resultados de las evaluaciones del 2012, hemos realizado modificaciones para el ciclo lectivo 2013, que permitan acercarnos más a la realización los objetivos propuestos para la asignatura. El éxito de esta mejora quedó evaluado en las encuestas del 2013, las cuales permiten que sigamos ajustando la propuesta pedagógica. La encuesta se divide en dos grandes áreas, aspectos organizativos y aspectos pedagógicos. En los primeros los estudiantes evalúan los aspectos del funcionamiento del Taller, la carga horaria, los espacios de trabajo grupal y lo plenarios. En cuanto a lo pedagógico se evalúan los contenidos propuestos en la asignatura a los fines de generar los procesos de integración. La encuesta comprendía preguntas de carácter cerrado en donde los estudiantes valoraban como "Muy Bueno", "Bueno", "Regular" o "Malo" aspectos vinculados a las grandes áreas antes mencionadas. También se dejó un espacio para observaciones, cuyo análisis fue de tipo cualitativo.

En base a los resultados obtenidos en el período lectivo 2012, se realizaron cambios sustanciales en la distribución de la carga horaria y en la organización del trabajo grupal en comisiones, favoreciendo la tarea docente a partir de grupos más reducidos. El trabajo en comisiones permitió durante el 2013 reforzar el contenido teórico, ya que se lo retomó al inicio y durante el intercambio entre estudiantes y docentes durante los trabajos prácticos. También se hizo mayor hincapié en trabajar las lecturas de bibliografía obligatoria en la clase práctica, lo 
que permitió reforzar los sustentos teóricos del taller. Los aspectos organizativos mejoraron sustancialmente en el 2013 (grafico 1), repercutiendo en la mejora del funcionamiento y comprensión del taller.

En cuanto a la percepción del Taller por los estudiantes de las encuestas realizadas se desprende que en su gran mayoría valoran el mismo, así como las modificaciones realizadas. Más del $95 \%$ consideraron al Taller como "bueno" o "muy bueno" en la satisfacción general en cuanto a conocimientos movilizados para el año 2013 (Grafico 2), siendo menor para el 2012. Los aspectos destacados se centran en la metodología de taller, que admite e incentiva la participación del estudiantado, otorgando el espacio y tiempo para la expresión, intercambio y debate de ideas y pensamientos diversos. La visualización y comprensión de la diversidad de sistemas productivos, productores, otros actores sociales intervinientes, y sus relaciones con el territorio, es otro de los aspectos rescatados.

Grafico 1. Resultados análisis comparativos encuestas final TIC II. Aspectos Organizativos. Año 2012 y 2013

Aspectos organizativos 2012

Satisfacción general

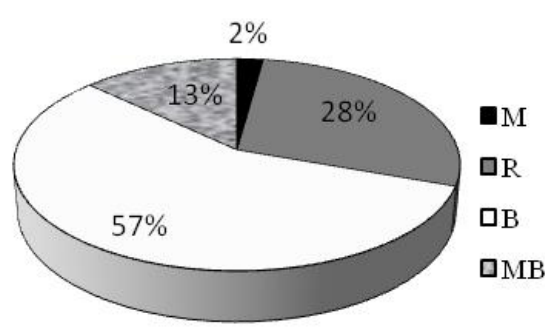

Aspectos organizativos 2013

Satisfacción general

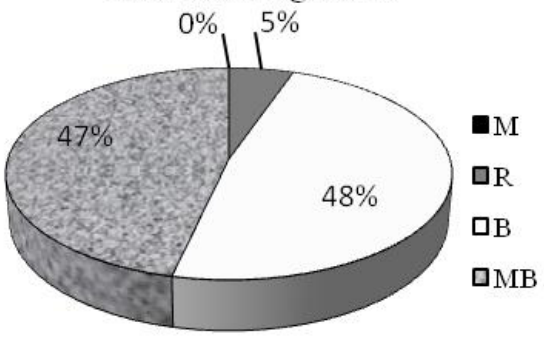

Otros ítems que fueron enunciados como observaciones en las encuestas de evaluación fueron la posibilidad de "acercarse a la realidad" y el "saberse profesionales"; y que el Taller otorga una "visión nueva" "integra lo social y lo productivo". 
Grafico 2. Resultados análisis comparativos encuestas final TIC II. Aspectos Pedagógicos. Año 2012 y 2013

Aspectos pedagógicos 2012

Satisfacción general



Aspectos pedagógicos 2013

Satisfacción general

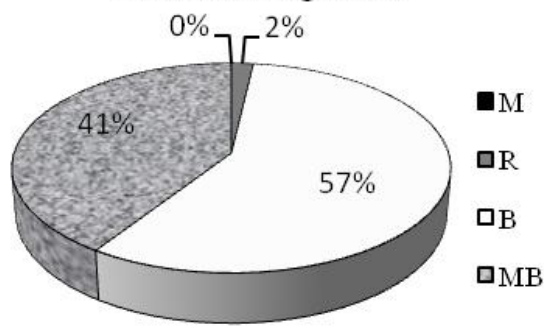

Por ultimo los estudiantes destacan el viaje de estudios realizado, siendo de los puntos más altos de las encuestas para ambos años (Grafico 3). El compartir diferentes situaciones a lo largo del viaje y la confección de los informes ha permitido a los estudiantes aprender a movilizar sus conocimientos adquiridos en situaciones muy disímiles. Así como en las encuestas se valora la posibilidad de integración desde los casos analizados a partir de testimonios, el viaje permite dar un cierre al taller, a partir del análisis y reflexión de las visitas a establecimientos productivos y el intercambio con actores vinculados a lo rural que se encuentran en la región visitada.

Grafico 3. Resultados análisis comparativos encuestas final TIC II. Viaje de Estudio. Año 2012 y 2013

Viaje de estudio 2012

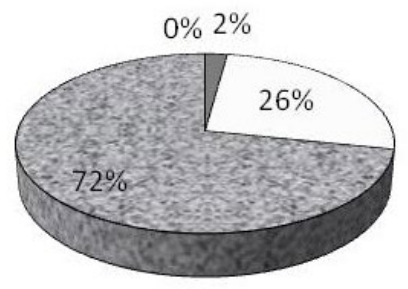

Viaje de estudio 2013

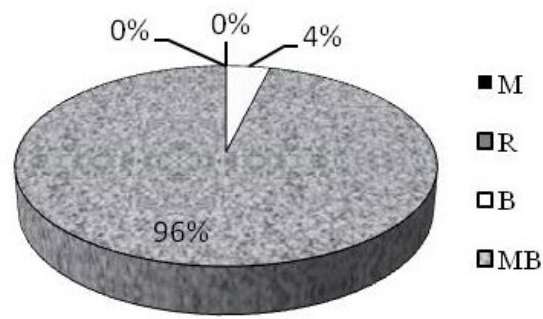




\section{Conclusiones}

La implementación de un Taller de integración curricular en una facultad generalista como la Facultad de Ciencias Agrarias y Forestales de la UNLP nos condujo a una visión renovada de la integración de los conocimientos.

Primero nos permitió dar cuenta de la diversidad y creciente diversificación de los modelos de desarrollo en el agro y en la actividad forestal, y consecuentemente la diversificación de los paradigmas socio-tecnológicos en los cuales deben intervenir nuestros futuros ingenieros forestales y agrónomos. La opción de la facultad es no dar orientación preferencial hacia uno de estos modelos, sino de encaminarse hacia ingenieros capaces de actuar en la complejidad de la realidad actual del mundo agropecuario y forestal.

Segundo, nos conduce a reanudar la tradición científica e intelectual de las ingenierías con un enfoque constructivista y en particular apto para modelizar la realidad en "contexto", noción que había sido borrada por las ciencias positivas. Lleva a dar un estatuto muy particular a la observación en contexto y entonces al valor pedagógico de la interacción con invitados que participan de distintos modelos de desarrollo y del viaje de estudio que se transforma en un viaje de integración.

Tercero, conduce al ingeniero a aprender a desarrollar una capacidad de ubicarse en un mundo indefinido en el cual, no sólo vale la capacidad demostrativa de sus conocimientos, sino también la capacidad argumentativa de los mismos: el ingeniero debe saber tomar una posición fundamentada en las ciencias, pero una posición que le es propia y que debe ser argumentada. La acción ya no puede ser vista como una simple aplicación de "la ciencia" sino una elección personal, profesionalmente fundamentada y argumentada en un mundo profesional donde prevalece el debate por sobre las recomendaciones.

Es así que, con el taller TIC II, estamos reinterpretando la carrera en función de una visión de nuestros estudiantes como ingenieros de una actividad territorializada. Las nociones de sistema, actores y territorio son centrales para la actividad de integración y permiten llegar a nuevos conceptos para la acción como es la de contexto, de planificación y de argumentación. En los años venideros debemos movilizar el TIC II como una plataforma de reflexión sobre las nuevas profesionalidades de nuestros ingenieros con los estudiantes y con los colegas de las otras disciplinas de las dos carreras. 


\section{Referencias}

Albaladejo, C; Bernal, M. C.; Cieza, R. Moreyra, A.; Goya, J; Senisterra, G.; Tamagno, L N.(2012) El viaje integrador para identificar los contextos de acción profesional. En IV Congreso Nacional y III Congreso Internacional de Enseñanza de las Ciencias Agropecuarias. La Plata. 9 al 11 de septiembre de 2012.

Albaladejo, C, Cieza, R; Moreyra, A. (2012) Repensar la ingeniería frente a la diversidad de paradigmas tecnológicos. La implementación de un curso de integración para las carreras de ingeniero agrónomo y forestal en la Universidad Nacional de La Plata. En Second World Engineering Education Forum (WEEF). Buenos Aires. Octubre de 2012.

Albaladejo, C. (2009). Médiations territoriales locales et développement rural. Vers de nouvelles compétences d'accompagnement de l'activité agricole. Les agricultures familiales dans les transformations territoriales en Argentine, au Brésil et en France, Université de Toulouse II Le Mirail. HDR Habilitation à Diriger des Recherches, Géographie et Aménagement: 304 p. Tesis de habilitación a Dirigir Doctorados.

Ander-Egg, E. (2003), Repensando la Investigación-Acción-Participativa: Comentarios, críticas y sugerencias. Grupo Editorial Lumen, Hvmanitas, Buenos Aires y México.

Arendt, H. (2004). La condición humana (introducción de Manuel Cruz). Buenos Aires, Paidós.366 páginas.

Brown, J.; Collins, A., and P. Duguid (1989), Situated Cognition and the Culture of Learning. Educational Researcher, 18 (1): 32-42

Díaz Maynard, Á. and R. Vellani (2008). Educación agrícola superior. Experiencias, ideas, propuestas. Montevideo, Uruguay, Universidad de la República de Uruguay y Comisión Sectorial de Enseñanza.

Gaignard, R. (1979). La Pampa argentine, l'occupation et la mise en valeur, Thèse d'Etat de l'Université de Bordeaux III. Doctorat d'Etat: 1174.

Hernández, V. (2007). Entrepreneurs "sans terre" et "pasteurs de la connaissance" : une nouvelle bourgeoisie rurale ? Turbulences monétaires et sociales: l'Amérique Latine dans une perspective comparée. V. Hernández, P. OuldAhmed, J. Papail and P. Phélinas. Paris, L'Harmattan: 209-257.

Hughes, E. C. (1963). “Profession.” Daedalus 92(4): 655-668. 
Laurent, C., M. Cerf, et al. (2002). "Le conseil en agriculture : un investissement immatériel entre développement sectoriel et développement territorial." Géographie, Economie, Société 2002(4): 131-153.

Le Moigne, J.-L. (1990). La théorie du système général. Théorie de la modélisation. Paris, P.U.F.

Moreyra, Alejandra (2009). Multiple Territories in Dispute: Water Policies, Participation and Mapuce Indigenous Rights in Patagonia Argentina. Tesis Doctoral disponible en http://search.library.wisc.edu/catalog/ocn489728046 / http://library.wur.nl/WebQuery/wda/lang/1908364

Röling, N. G. (1992). Agricultural knowledge and information systems: Models for knowledge management. Processes and practices for change Professionals. D. J. Blackburn. Guelph, Ontario, University of Guelph: 29. 